\title{
Antimicrobial susceptibility of Acinetobacter baumannii isolated from hospital patients
}

\author{
Wanutsanun Tunyapanit $^{\mathrm{a}, *}$, Pornpimol Pruekprasert ${ }^{\mathrm{a}}$, Kamolwish Laoprasopwattana ${ }^{\mathrm{a}}$, \\ Sureerat Chelae $^{\mathrm{b}}$ \\ a Department of Pediatrics, Faculty of Medicine, Prince of Songkla University, Hat Yai, \\ Songkhla 90110 Thailand \\ b Department of Pathology, Faculty of Medicine, Prince of Songkla University, Hat Yai, \\ Songkhla 90110 Thailand \\ *Corresponding author, e-mail: wanutsanun.t@psu.ac.th
}

Received 26 Sep 2012

Accepted 31 Oct 2013

\begin{abstract}
The incidence of multidrug-resistant Acinetobacter baumannii (MDR A. baumannii) is increasing worldwide and is leading to therapeutic problems. We investigated the in vitro activities of cefoperazone/sulbactam, colistin, imipenem, and rifampicin alone and in double combinations against $100 \mathrm{~A}$. baumannii isolates from patients at Songklanagarind Hospital in Songkhla Province, Thailand. The E-test method was used to determine antimicrobial susceptibility, the minimal inhibitory concentration (MIC) and for antimicrobial combination testing. A. baumannii isolates were susceptible to colistin (97\%), cefoperazone/sulbactam (69\%), imipenem (45\%), and rifampicin (13\%). Fifty-nine percent of them were MDR A. baumannii. Colistin was superior to cefoperazone/sulbactam, rifampicin and imipenem against MDR A. baumannii and the $\mathrm{MIC}_{50}, \mathrm{MIC}_{90}$ of colistin were 0.75 and $1 \mu \mathrm{g} / \mathrm{ml}$, respectively. Non-MDR A. baumannii isolates were susceptible to cefoperazone/sulbactam (100\%), colistin (95\%), imipenem (93\%) and rifampicin (2\%). Combinations of cefoperazone/sulbactam plus colistin or rifampicin, imipenem plus colistin or rifampicin and colistin plus rifampicin showed indifferent effects against most MDR isolates. Of all the antimicrobial combinations tested, cefoperazone/sulbactam plus rifampicin produced the highest percentages (42\%) of synergy, partial synergy, and additive results. The activity rate of cefoperazone/sulbactam against MDR A. baumannii was higher when combined with rifampicin than colistin. Thus colistin had the greatest activity against most MDR and non-MDR A. baumannii isolates among all of the antibiotics tested. Cefoperazone/sulbactam and imipenem showed good activity against non-MDR isolates, and cefoperazone/sulbactam combined with rifampicin may be useful in treating infections caused by MDR isolates.
\end{abstract}

KEYWORDS: drug combinations, multidrug-resistant A. baumannii, colistin, cefoperazone/sulbactam, imipenem, rifampicin

\section{INTRODUCTION}

A. baumannii is one of the most important nosocomial pathogens because of its longevity in the hospital environment and ability to resist various antimicrobial agents, such as resistance to broad-spectrum $\beta$-lactam antibiotics by $\beta$-lactamases production. Changes in penicillin-binding proteins prevent their action resulting in penicillin resistance. Alterations in the structure and number of porin protein result in decreased permeability to antibiotics through the outer membrane of the bacterial cell. The activity of efflux pumps lead to a reduction of antibiotic concentration within the bacterial cell. Resistance to aminoglycoside antibiotics results from aminoglycoside-modifying enzymes, AdeABC efflux pump, and 16S rRNA methylation. Modifications in the structure of DNA gyrase (mutations in the gyrA and parC genes) reduce the biding affinity of quinolones to the enzyme-DNA complex resulting in fluoroquinolone antibiotics resistance $^{1}$. A. baumannii frequently causes ventilatorassociated pneumonia, urinary tract infection, meningitis, surgical site infection, and bacteraemia ${ }^{1}$.

Multidrug-resistant A. baumannii (MDR A. baumannii) is resistant to more than two antimicrobial classes such as antipseudomonal cephalosporins, antipseudomonal carbapenems, $\beta$-lactam/ $\beta$-lactamase inhibitors, aminoglycosides, and fluoroquinolones.

The incidence of MDR A. baumannii is increasing worldwide, including Europe, North America, Latin America, and Asia ${ }^{1}$. Infections caused by MDR A. baumannii are associated with high morbidity rates, especially in immunocompromised patients, patients admitted to intensive care units, and patients treated 
with broad-spectrum antibiotics ${ }^{1,2}$.

Antimicrobial agents such as imipenem, sulbactam, colistin and rifampicin have been used for A. baumannii treatment ${ }^{1,3}$. However, colistin-resistant and imipenem-resistant $A$. baumannii have emerged and these isolates are often multidrug-resistant ${ }^{1,4,5}$.

Previous in vitro studies have demonstrated that a combination of antimicrobial agents such as colistin and rifampicin, colistin and imipenem, imipenem and rifampicin, and cefoperazone/sulbactam combined with imipenem produce better activity against

A. baumannii and MDR A. baumannii ${ }^{6-8}$. The combination of antimicrobial agents seems to be an alternative in A. baumannii treatment.

The aim of this study was to determine the in vitro activity of cefoperazone/sulbactam, colistin, imipenem, and rifampicin alone and in double combinations against $A$. baumannii isolated from patients at Songklanagarind Hospital in Southern Thailand.

\section{MATERIALS AND METHODS}

\section{Bacterial strains}

One hundred A. baumannii isolates were collected from clinical specimens such as sputum (64), blood (12), urine (8), pus (from leg, 2; pleural empyema, 1; axilla, 2; vagina, 1; percutaneous endoscopic gastrostomy tube, 1; and eye, 1), body fluid (6) and tissue (2) from patients at Songklanagarind Hospital in Songkhla Province, Thailand during the JanuaryJuly 2010 period. Each isolate was collected from a different patient. Bacterial isolation and identification were performed using standard laboratory methods ${ }^{9}$. This study was approved by the Ethics Committee of the Faculty of Medicine, Prince of Songkla University (EC 53-139-01-7-3-1).

\section{MDR A. baumannii detection}

Antimicrobial susceptibilities to amikacin (30 $\mu \mathrm{g}$, AK), cefoperazone/sulbactam (30/75 $\mu \mathrm{g}$, SPZ), ceftazidime (30 $\mu \mathrm{g}, \mathrm{CAZ})$, ciprofloxacin (5 $\mu \mathrm{g}, \mathrm{CIP})$ and imipenem (10 $\mu \mathrm{g}$, IMP) (Oxoid, Ltd., Basingstoke, Hampshire, England) were determined by disk diffusion as recommended by the Clinical and Laboratory Standards Institute (CLSI) guidelines ${ }^{10}$. MDR A. baumannii was defined as an intermediately-resistant or resistant isolate to more than two of the following five antimicrobial agents: amikacin, cefoperazone/sulbactam, ceftazidime, ciprofloxacin, and imipenem ${ }^{1}$.

\section{Antimicrobial susceptibility testing and MIC determination}

The E-test was used to determine antimicrobial susceptibility and the minimal inhibitory concen- tration (MIC) of cefoperazone/sulbactam (0.016$256 \mu \mathrm{g} / \mathrm{ml}$, CPS $)$, colistin $(0.016-256 \mu \mathrm{g} / \mathrm{ml}, \mathrm{CO})$, imipenem $(0.002-32 \mu \mathrm{g} / \mathrm{ml}$, IP), and rifampicin (0.002-32 $\mu \mathrm{g} / \mathrm{ml}, \mathrm{RI})$ (AB Biodisk, Solna, Sweden) against $A$. baumannii based on CLSI guidelines ${ }^{10}$. The cefoperazone MIC breakpoint was used for cefoperazone/sulbactam ${ }^{11}$. Rifampicin MICs of $\leqslant 2 \mu \mathrm{g} / \mathrm{ml}$ were considered as susceptible, MICs of $4-8 \mu \mathrm{g} / \mathrm{ml}$ were considered as low-level resistant, and MICs of up to $256 \mu \mathrm{g} / \mathrm{ml}$ were considered as high-level resistant $^{12,13}$. Escherichia coli (E. coli) ATCC 25922 was used as a control strain.

\section{Antimicrobial combination testing}

The antimicrobial combination testing was carried out via the E-test method ${ }^{14}$. Briefly, E-test strips of the two antimicrobial agents were placed at an angle of $90^{\circ}$ at the MIC of each antimicrobial agent on an inoculated Mueller Hinton agar plate. The plates were incubated at $35^{\circ} \mathrm{C}$ for $18-24 \mathrm{~h}$. The inhibition zone of each antimicrobial agent intersecting the E-test strip was interpreted as the MIC in combination. Fractional inhibitory concentrations (FICs) were calculated by dividing the MIC of drug $\mathrm{A}$ and $\mathrm{B}$ in combination by the MIC of drug A or B alone. The fractional inhibitory concentration index (FICI) was obtained by the sum of the FICs of each drug. The FICI was interpreted as follows: FICI $\leqslant 0.5$ represented synergy, $0.5<$ FICI $<1$ represented partial synergy, FICI $=1$ represented additive effects, $1<$ FICI $<4$ represented indifference, and FICI $\geqslant 4$ represented $\operatorname{antagonism}^{14,15}$.

\section{RESULTS}

\section{Antimicrobial susceptibility}

The antimicrobial susceptibility of $100 \mathrm{~A}$. baumannii isolates against four antimicrobial agents is shown in Table 1. Among all the antimicrobial agents tested, $97 \%, 69 \%, 45 \%$, and $13 \%$ of isolates were susceptible to colistin, cefoperazone/sulbactam, imipenem, and rifampicin, respectively. The $\mathrm{MIC}_{50}$ and $\mathrm{MIC}_{90}$ values of these four antimicrobial agents showed that most isolates were susceptible to colistin; it had the highest antimicrobial activity. Cefoperazone/sulbactam provided moderate activity, with an $\mathrm{MIC}_{50}$ of $12 \mu \mathrm{g} / \mathrm{ml}$. The $\mathrm{MIC}_{50}$ and $\mathrm{MIC}_{90}$ of imipenem were higher than the CLSI susceptibility breakpoint, indicating that imipenem had a low antimicrobial activity against these isolates. The $\mathrm{MIC}_{50}$ and $\mathrm{MIC}_{90}$ of rifampicin were $4 \mu \mathrm{g} / \mathrm{ml}$ and $6 \mu \mathrm{g} / \mathrm{ml}$, respectively, indicating that A. baumannii possesses a low-level resistance against this antimicrobial agent. 
Table 1 The $\mathrm{MIC}_{50}$ and $\mathrm{MIC}_{90}$ values, and the percentages of susceptibility rate of $100 \mathrm{~A}$. baumannii isolates against 4 antimicrobial agents.

\begin{tabular}{|c|c|c|c|c|c|c|c|c|c|}
\hline \multirow[t]{3}{*}{ Antimicrobial agents } & \multirow{2}{*}{\multicolumn{3}{|c|}{ A. baumannii $(n=100)$}} & \multicolumn{6}{|c|}{ Antimicrobial susceptibility } \\
\hline & & & & \multicolumn{3}{|c|}{ Non-MDR $(n=41)$} & \multicolumn{3}{|c|}{$\operatorname{MDR}(n=59)$} \\
\hline & $\mathrm{MIC}_{50}$ & $\mathrm{MIC}_{90}$ & $\mathrm{~S}(\%)$ & $\mathrm{MIC}_{50}$ & $\mathrm{MIC}_{90}$ & $\mathrm{~S}(\%)$ & $\mathrm{MIC}_{50}$ & $\mathrm{MIC}_{90}$ & $\mathrm{~S}(\%)$ \\
\hline Colistin & 0.75 & 1 & $97(97)$ & 1 & 1.5 & $39(95)$ & 0.75 & 1 & $58(98)$ \\
\hline Cefoperazone/sulbactam & 12 & 64 & $69(69)$ & 1.5 & 2 & $41(100)$ & 24 & 96 & $28(47)$ \\
\hline Imipenem & 16 & $>32$ & $45(45)$ & 0.19 & 0.5 & $38(93)$ & $>32$ & $>32$ & $7(12)$ \\
\hline Rifampicin & 4 & 6 & $13(13)$ & 4 & 8 & $1(2)$ & 4 & 6 & $12(20)$ \\
\hline
\end{tabular}

MIC breakpoints of cefoperazone/sulbactam: susceptible $\leqslant 16 \mu \mathrm{g} / \mathrm{ml}$, intermediately-resistant $32 \mu \mathrm{g} / \mathrm{ml}$, resistant $\geqslant$ $64 \mu \mathrm{g} / \mathrm{ml}$; colistin: susceptible $\leqslant 2 \mu \mathrm{g} / \mathrm{ml}$, resistant $\geqslant 4 \mu \mathrm{g} / \mathrm{ml}$; imipenem: susceptible $\leqslant 4 \mu \mathrm{g} / \mathrm{ml}$, intermediatelyresistant $8 \mu \mathrm{g} / \mathrm{ml}$, resistant $\geqslant 16 \mu \mathrm{g} / \mathrm{ml}$; rifampicin: susceptible $\leqslant 2 \mu \mathrm{g} / \mathrm{ml}$, low-level resistant $4-8 \mu \mathrm{g} / \mathrm{ml}$ and high-level resistant $\geqslant 256 \mu \mathrm{g} / \mathrm{ml}$ (CLSI, 2011)

The results of antimicrobial susceptibility tests showed that $59 \%$ of isolates were MDR A. baumannii. Yet, both MDR and non-MDR A. baumannii were susceptible to colistin (98\% and $95 \%$, respectively) and had $\mathrm{MIC}_{50}$ and $\mathrm{MIC}_{90}$ values $<2 \mu \mathrm{g} / \mathrm{ml}$. All the non-MDR A. baumannii were highly susceptible to cefoperazone/sulbactam with $\mathrm{MIC}_{50}$ and $\mathrm{MIC}_{90}$ values of $\leqslant 2 \mu \mathrm{g} / \mathrm{ml}$, thirty-eight $(93 \%)$ isolates were susceptible to imipenem and had $\mathrm{MIC}_{50}$ and $\mathrm{MIC}_{90}$ values of $\leqslant 0.5 \mu \mathrm{g} / \mathrm{ml}$, while only one $(2 \%)$ isolate was susceptible to rifampicin, having $\mathrm{MIC}_{50}$ and $\mathrm{MIC}_{90}$ values of $4 \mu \mathrm{g} / \mathrm{ml}$ and $8 \mu \mathrm{g} / \mathrm{ml}$, respectively. Twenty-eight (47\%) of the MDR A. baumannii were susceptible to cefoperazone/sulbactam. The corresponding $\mathrm{MIC}_{50}$ and $\mathrm{MIC}_{90}$ values were $24 \mu \mathrm{g} / \mathrm{ml}$ and $96 \mu \mathrm{g} / \mathrm{ml}$, respectively. Only seven (12\%) isolates were susceptible to imipenem and had $\mathrm{MIC}_{50}$ and $\mathrm{MIC}_{90}$ values of $>32 \mu \mathrm{g} / \mathrm{ml}$, and twelve $(20 \%)$ isolates were susceptible to rifampicin, having $\mathrm{MIC}_{50}$ and $\mathrm{MIC}_{90}$ values of $4 \mu \mathrm{g} / \mathrm{ml}$ and $6 \mu \mathrm{g} / \mathrm{ml}$, respectively.

\section{Antimicrobial combination}

The effects of double combinations of cefoperazone/sulbactam, colistin, imipenem and rifampicin against A. baumannii isolates are shown in Table 2 .

Among all the antimicrobial combinations tested, an indifferent effect was observed in most MDR isolates. Nevertheless, synergy, partial synergy and additive effects could be detected in some isolates. The results indicated that synergy, partial synergy and additive effects were $0-7 \%, 7-24 \%$, and $0-15 \%$, respectively.

Of the antimicrobial combinations tested, cefoperazone/sulbactam plus rifampicin produced the highest percentages $(42 \%)$ of the total of synergy, partial synergy, and additive results. Only one MDR A. baumannii isolate revealed an antagonistic effect for the cefoperazone/sulbactam plus colistin and cefoperazone/sulbactam plus rifampicin combinations.

The percentages of MDR A. baumannii isolates susceptible to cefoperazone/sulbactam (47\%) increased when it was combined with rifampicin $(66 \%)$ or colistin (59\%). Additionally, the $\mathrm{MIC}_{50}$ of cefoperazone/sulbactam against MDR A. baumannii isolates $(24 \mu \mathrm{g} / \mathrm{ml})$ was lowered when it was combined with rifampicin $(16 \mu \mathrm{g} / \mathrm{ml})$ or colistin $(16 \mu \mathrm{g} / \mathrm{ml})$. Imipenem combined with rifampicin or colistin and imipenem alone showed poor activity against MDR isolates; the percentages of susceptibility rates were $13 \%, 13 \%$ and $12 \%$, respectively. The $\mathrm{MIC}_{50}$ values of imipenem against MDR A. baumannii were $>32 \mu \mathrm{g} / \mathrm{ml}$. When imipenem and rifampicin or imipenem and colistin were used together, the $\mathrm{MIC}_{50}$ values of imipenem in combinations were higher than the CLSI susceptibility breakpoint. The results demonstrated that combining imipenem and rifampicin reduced the $\mathrm{MIC}_{50}$ of imipenem from $>32$ to $24 \mu \mathrm{g} / \mathrm{ml}$, and the combination of imipenem and colistin lowered the $\mathrm{MIC}_{50}$ of imipenem from $>32$ to $32 \mu \mathrm{g} / \mathrm{ml}$ (Table 3 ).

\section{DISCUSSION}

The in vitro antimicrobial susceptibility test results of this study reveal that colistin possesses a higher activity rate against $A$. baumannii than cefoperazone/sulbactam, imipenem, or rifampicin. Similar data obtained from studies from Chiang Mai University Hospital in Northern Thailand indicate that colistin has a higher activity against $A$. baumannii than cefoperazone/sulbactam or imipenem (out of $132 \mathrm{~A}$. baumannii isolates, $96 \%, 77 \%$, and $64 \%$ were susceptible to colistin, cefoperazone/sulbactam, and imipenem, respectively $)^{16}$. The result of clinical effectiveness of colistin monotherapy and combination therapy suggested that colistin alone achieves cure and/or 
Table 2 Effect of double combinations of cefoperazone/sulbactam (CPS), colistin (CO), imipenem (IP), and rifampicin (RI) against 59 MDR A. baumannii isolates.

\begin{tabular}{lcccccc}
\hline $\begin{array}{l}\text { Antimicrobial } \\
\text { combinations }\end{array}$ & Synergy & $\begin{array}{c}\text { Partial } \\
\text { synergy }\end{array}$ & $\begin{array}{c}\text { Additive } \\
\text { effects }\end{array}$ & $\begin{array}{c}\text { Total synergy, partial synergy } \\
\text { and additive effects }\end{array}$ & Indifference & Antagonism \\
\cline { 2 - 7 } & $4(7)$ & $10(17)$ & $1(2)$ & $15(26)$ & $43(73)$ & $1(2)$ \\
CPS + CO & $2(3)$ & $14(24)$ & $9(15)$ & $25(42)$ & $33(56)$ & $1(2)$ \\
CPS + RI & $3(5)$ & $6(10)$ & $0(0)$ & $9(15)$ & $50(85)$ & $0(0)$ \\
IP + CO & $1(2)$ & $4(7)$ & $2(3)$ & $7(12)$ & $52(88)$ & $0(0)$ \\
IP + RI & $0(0)$ & $4(7)$ & $2(3)$ & $6(10)$ & $53(90)$ & $0(0)$ \\
CO + RI &
\end{tabular}

Fractional inhibitory concentration index (FICI): synergy, FICI $\leqslant 0.5$; partial synergy, $0.5<$ FICI $<1$; additive effect, FICI $=1$; indifference, $1<$ FICI $<4$; antagonism, FICI $\geqslant 4$.

Table 3 The $\mathrm{MIC}_{50}$ values and the percentages of the susceptibility rate of 59 MDR A. baumannii isolates against a single antimicrobial agent and antimicrobial agent combinations.

\begin{tabular}{lcc}
\hline Antimicrobial agents & $\mathrm{MIC}_{50}(\mu \mathrm{g} / \mathrm{ml})$ & $\mathrm{S}(\%)$ \\
\hline $\mathrm{CPS}^{*}$ & 24 & $28(47)$ \\
$\mathrm{CPS}^{*}+\mathrm{CO}$ & 16 & $35(59)$ \\
$\mathrm{CPS}^{*}+\mathrm{RI}$ & 16 & $39(66)$ \\
$\mathrm{IP}^{*}$ & $>32$ & $7(12)$ \\
$\mathrm{IP}^{*}+\mathrm{CO}$ & 32 & $8(13)$ \\
$\mathrm{IP}^{*}+\mathrm{RI}$ & 24 & $8(13)$ \\
\hline
\end{tabular}

* Main drug.

MIC breakpoints of cefoperazone/sulbactam: susceptible $\leqslant 16 \mu \mathrm{g} / \mathrm{ml}$ and imipenem: susceptible $\leqslant 4 \mu \mathrm{g} / \mathrm{ml}$ (CLSI, 2011).

improvement rates ranging from $57 \%$ to $78 \% 17,18$, whereas the equivalent rates for combination therapy showed $67 \%$ to $74 \%{ }^{18}$.

Colistin monotherapy has shown the problem of nephrotoxicity, neurotoxicity, colistin-resistance, and heteroresistance among gram-negative bacterial populations ${ }^{19-21}$. Nephrotoxicity is a major concern when colistin is administered in patients with a history of chronic renal failure ${ }^{17}$. Colistin combination therapy is being strongly recommended against monotherapy due to selection of heteroresistant strains during prolonged colistin therapy ${ }^{22}$ and heteroresistance strains were reported to be correlated with endemic infections in $\mathrm{ICU}^{23}$. Thus combination therapy might be beneficial for clinicians to prevent the emergence of resistance during therapy, decreased nephrotoxicity, especially if therapeutic options are limited.

The antimicrobial resistance studies of $A$. baumannii in Thailand during the 2000-2005 period revealed a $46-56 \%$ resistance rate by MDR $A$. baumannii isolates ${ }^{24}$. Our results indicated that $59 \%$ of
A. baumannii isolates were MDR. Furthermore, both MDR and non-MDR A. baumannii isolates remained highly susceptible to colistin. Various reports from many countries, such as China, Greece, and Turkey have shown $21.5-84 \%$ of $A$. baumannii isolates during 1996-2009 were MDR A. baumannii and 94-100\% of these isolates were susceptible to colistin ${ }^{11,25,26}$. Ninety-eight percent of MDR A. baumannii isolates were susceptible to colistin and had $\mathrm{MIC}_{50}$ and $\mathrm{MIC}_{90}$ values of 0.75 and $1 \mu \mathrm{g} / \mathrm{ml}$, which were similar to those of previous studies from Dizbay ${ }^{7,8,25}$. This indicates that colistin was effective antimicrobial agent against MDR A. baumannii.

Imipenem and cefoperazone/sulbactam have generally been used in A. baumannii treatment ${ }^{4,24}$. Our data also affirm that cefoperazone/sulbactam and imipenem are good choices for the eradication of nonMDR A. baumannii isolates.

Moreover, rifampicin has been proposed as an alternative antimicrobial agent for the treatment of MDR A. baumannii, based on outcomes of in vitro studies $^{2,7,8}$ and in vivo infection models ${ }^{12,27}$. A previous study has shown that $64 \%$ of MDR A. baumannii were susceptible to rifampicin ${ }^{7}$. In contrast, our results show that rifampicin has low antimicrobial activity against most MDR and non-MDR A. baumannii. As with our findings, the study by GiamarellosBourboulis et al reported that $15 \%$ of MDR A. baumannii were susceptible to rifampicin, having $\mathrm{MIC}_{50}$ and $\mathrm{MIC}_{90}$ values higher than $2 \mu \mathrm{g} / \mathrm{ml}^{3}$.

The combination antimicrobial therapy has been used as an alternative in MDR A. baumannii treatment. The presence of synergy, partial synergy, and additive effect could potentially reduce toxicity and improve outcomes for patients with difficult-to- treat infections $^{28}$. Forty-seven of 59 MDR A. baumannii isolates in this study $(80 \%)$ were resistance to rifampicin (45 isolates had MIC ranges 3-8 $\mu \mathrm{g} / \mathrm{ml}$ 
and 2 isolates had MICs $>32 \mu \mathrm{g} / \mathrm{ml}$ ). The synergy, partial synergy, and additive results could be detected in 28 isolates in the case of susceptible (1 isolate) and resistance to rifampicin (27 isolates).

We found that 1 of 12 MDR A. baumannii isolates was susceptible to rifampicin revealed synergy result for cefoperazone/sulbactam plus rifampicin and imipenem plus rifampicin. Twenty-seven of $45 \mathrm{MDR}$ A. baumannii isolates were resistance to rifampicin (MIC ranges 3-8 $\mu \mathrm{g} / \mathrm{ml}$ ); 16 isolates revealed synergy, partial synergy, and additive results for cefoperazone/sulbactam plus rifampicin, 5 isolates revealed partial synergy and additive results for imipenem plus rifampicin, 5 isolates revealed partial synergy and additive results for cefoperazone/sulbactam plus rifampicin and colistin plus rifampicin, 1 isolate revealed partial synergy and additive results for cefoperazone/sulbactam plus rifampicin, imipenem plus rifampicin and colistin plus rifampicin. Although Rifampicin is a hydrophobic antibiotic, its negative charge and large molecular size cause it to be unable to effectively penetrate through the outer membrane of A. baumannii alone ${ }^{5}$. Other antibiotics which combine with rifampicin may be related to substantial changes in the outer membrane of A. baumannii isolates; thus enhancing the ability of rifampicin to penetrate into the cell ${ }^{29}$. The effectiveness of rifampicin in antimicrobial combinations, however, could depend on the degree of sensitivity and specific resistance mechanisms of individual A. baumannii isolates ${ }^{30}$.

Previously, the efficacy of rifampicin in association with antimicrobial agent was demonstrated even in the case of strains susceptible and resistance to rifampicin $^{3,7}$. In colistin combined with rifampicin, Timurkaynak et al found that four MDR A. baumannii isolates ( 3 of 4 isolates were susceptible to rifampicin and 1 of 4 isolates were low-level resistant to rifampicin) exhibited synergy effect and one MDR A. baumannii isolates was resistance to rifampicin showed partial synergy effect ${ }^{7}$. The study of Giamarellos-Bourboulis et $\mathrm{al}^{3}$ using the time-kill method showed $85 \%$ of MDR A. baumannii isolates were resistant to rifampicin, having MIC ranges of 2$64 \mu \mathrm{g} / \mathrm{ml}$. The combination of colistin and rifampicin was a synergy effect against 15\% of MDR A. baumannii isolates ${ }^{3}$.

Combinations of rifampicin with ampicillin/sulbactam, imipenem with colistin or rifampicin, and colistin with rifampicin have shown promising results against MDR A. baumannii ${ }^{6-8}$. Recently, Ozseven et al found that the combination of imipenem plus rifampicin produced a $73 \%$ rate of synergy effect against 34 MDR A. baumannii isolates evaluated by the chequerboard microdilution method ${ }^{31}$. Likewise, Pongpech et al reported that the synergy effect of imipenem plus colistin and sulbactam plus colistin against 30 MDR A. baumannii isolates by the chequerboard microdilution panel method were $100 \%$ and $53 \%$, respectively ${ }^{32}$. Furthermore, the study of Timurkaynak et al reported the synergy effect of colistin plus rifampicin against 4 MDR A. baumannii isolates by the chequerboard method at $100 \%{ }^{7}$. In this study, all the antimicrobial combinations tested by the E-test method yielded a predominantly indifferent effect.

Our findings indicate that the synergy effects of cefoperazone/sulbactam combined with colistin or rifampicin, imipenem combined with colistin or rifampicin, and colistin combined with rifampicin were lower than the results of previous studies investigating the same in vitro synergy effect by the chequerboard $\operatorname{method}^{7,31,32}$. However, the effects of antimicrobial combinations against organisms could depend on individual strains and evaluation methods ${ }^{6}$. Nevertheless, our study demonstrated that the activity of cefoperazone/sulbactam against MDR A. baumannii could be improved by combining it with rifampicin. This suggests that cefoperazone/sulbactam plus rifampicin may be a reasonable choice in the treatment of infections by these organisms. A. baumannii is the most common bacteria causing nosocomial pneumonia. Although MDR A. baumannii is highly susceptible to colistin, the clinical response rate to this antibiotic is only $25-62 \%$ due to low colistin levels in lungs tissue $^{33}$. In addition, the main adverse effects of colistin are nephrotoxic and neurotoxic. The incidences of renal toxicity and neurotoxicity are $7-69 \%^{20,21}$ and $7 \%{ }^{34}$, respectively.

We suggest that, in patients suffering from MDR A. baumannii nosocomial pneumonia that is not responsive to colistin, the combination of cefoperazone/sulbactam plus rifampicin might be beneficial. However, the combination of cefoperazone/sulbactam with rifampicin needs to be studied on a larger scale than that afforded by this investigation.

We did not use the standard testing method (chequerboard method and time-kill test) for evaluated the in vitro synergy activity of antimicrobial combination against $A$. baumannii isolates. The synergy results in this study may be insensitive to the E-test method when compared with the chequerboard and time-kill test. The agreement between chequerboard method and E-test was $63 \%$ and between time-kill test and E-test method was $72 \%$, the minor disagreements occurred when synergy was observed using either the chequerboard or time-kill test while additive or 
indifference was observed using the E-test method $^{22}$. In a combination therapy for A. baumannii isolates, therefore, interpretation of results requires caution in empirical therapy.

In conclusion, the findings of this study indicate that colistin has the best activity against $A$. baumannii, whereas cefoperazone/sulbactam and imipenem are good choices for the treatment of non-MDR A. baumannii infections.

Acknowledgements: This work was supported by a grant from the Faculty of Medicine, Prince of Songkla University. We thank all the participating patients for providing the specimens and the microbiology personnel of the Department of Pathology, Faculty of Medicine, Prince of Songkla University for providing the A. baumannii isolates. Our gratitude also goes to Mrs Lamy Kaewjungwad for providing and assisting with the laboratory facilities, and Mr Edmond Subashi for help with correcting the English.

\section{REFERENCES}

1. Peleg AY, Seifert H, Paterson DL (2008) Acinetobacter baumannii: emergence of a successful pathogen. Clin Microbiol Rev 21, 538-82.

2. Neonakis IK, Spandidos DA, Petinaki E (2011) Confronting multidrug-resistant Acinetobacter baumannii: a review. Int J Antimicrob Agents 37, 102-9.

3. Giamarellos-Bourboulis EJ, Xirouchaki E, Giamarellou H (2001) Interactions of colistin and rifampin on multidrug-resistant Acinetobacter baumannii. Diagn Microbiol Infect Dis 40, 117-20.

4. Jamulitrat S, Thongpiyapoom S, Suwalak N (2007) An outbreak of imipenem-resistant Acinetobacter baumannii at Songklanagarind Hospital: the risk factors and patient prognosis. J Med Assoc Thai 90, 2181-91.

5. Li J, Nation RL, Owen RJ, wong S, Spelman D, Franklin C (2007) Antibiograms of multidrug-resistant clinical Acinetobacter baumannii: promising therapeutic options for treatment of infection with colistinresistant strains. Clin Infect Dis 45, 594-8.

6. Kiratisin P, Apisarnthanarak A, Kaewdaeng S (2010) Synergistic activities between carbapenems and other antimicrobial agents against Acinetobacter baumannii including multidrug-resistant and extensively drugresistant isolates. Int J Antimicrob Agents 36, 243-6.

7. Timurkaynak F, Can F, Azap OK, Demirbilek M, Arslan H, Karaman SO (2006) In vitro activities of non-traditional antimicrobials alone or in combination against multidrug-resistant strains of Pseudomonas aeruginosa and Acinetobacter baumannii isolated from intensive care units. Int J Antimicrob Agents 27, 224-8.

8. Tripodi MF, Durante-Mangoni E, Fortunato R, Utili $\mathrm{R}$, Zarrilli R (2007) Comparative activities of colistin, rifampicin, imipenem and sulbactam/ampicillin alone or in combination against epidemic multidrug-resistant
Acinetobacter baumannii isolates producing OXA-58 carbapenemases. Int J Antimicrob Agents 30, 537-40.

9. Von Graevenitz A (1995) Acinetobacter, Alcaligenes, Moraxella and other nonfermentative Gram-negative bacteria. In: Murray PR, Baron EJ, Pfaller MA, Tenover FC, Yolken RH (eds) Manual of Clinical Microbiology, 6th edn, American Society for Microbiology, Washington D.C., pp 520-32.

10. Clinical Laboratory Standards Institute (2011) Performance standards for antimicrobial susceptibility testing; 21st informational supplement, CLSI M100-S21, Clinical and Laboratory Standards Institute Wayne, PA, U.S.A.

11. Wang H, Chen M (2005) Surveillance for antimicrobial resistance among clinical isolates of gram-negative bacteria from intensive care unit patients in China, 1996 to 2002. Diagn Microbiol Infect Dis 51, 201-8.

12. Saballs M, Pujol M, Tubau F, Peña $C$, Montero A, Domínguez MA, Gudiol F, Ariza J (2006) Rifampicin/imipenem combination in the treatment of carbapenem-resistant Acinetobacter baumannii infections. J Antimicrob Chemother 58, 697-700.

13. Tan TY, Ng LSY, Poh K (2007) Susceptibility testing of unconventional antibiotics against multiresistant Acinetobacter spp. by agar dilution and Vitek2. Diagn Microbiol Infect Dis 58, 357-61.

14. White RL, Burgess DS, Manduru M, Bosso JA (1996) Comparison of three different in vitro methods of detecting synergy: time-kill, checkerboard, and E test. Antimicrob Agents Chemother 40, 1914-8.

15. Kiffer CRV, Sampaio JLM, Sinto S, Oplustil CP, Koga PCM, Arruda AC, Turner PJ, Mendes C (2005) In vitro synergy test of meropenem and sulbactam against clinical isolates of Acinetobacter baumannii. Diagn Microbiol Infect Dis 52, 317-22.

16. Leepethacharat K, Oberdorfer P (2007) Acinetobacter baumannii infection and colonization among pediatric patients at Chiang Mai University Hospital. J Infect Dis Antimicrob Agents 24, 63-73.

17. Michalopoulos AS, Tsiodras S, Rellos K, Mentzelopoulos S, Falagas ME (2005) Colistin treatment in patients with ICU-acquired infections caused by multiresistant gram-negative bacteria: the renaissance of an old antibiotic. Clin Microbiol Infect 11, 115-21.

18. Petrosillo N, Ioannidou E, Falagas ME (2008) Colistin monotherapy versus combination therapy: evidence from microbiological, animal and clinical studies. Clin Microbiol Infect 14, 816-27.

19. Li Jian Rayner CR, Nation RL, Owen RJ, Spelman D, Tan KE, Liolios L (2006) Heteroresistance to colistin in multidrug-resistant Acinetobacter baumannii. Antimicrob Agents Chemother 50, 2946-50.

20. Falagas ME, Kasiakou SK (2005) Colistin: the revival of polymyxins for the management of multidrugresistant gram-negative bacterial infections. Clin Infect Dis 4, 1333-41. 
21. Pogue JM, Lee J, Marchaim D, Yee V, Zhao JJ, Chopra T, Lephart P, Kaye KS (2011) Incidence of and risk factors for colistin-associated nephrotoxicity in a large academic health system. Clin Infect Dis 53, 879-84.

22. David MD, Gill MJ (2008) Potential for underdosing and emergence of resistance in Acinetobacter baumannii during treatment with colistin. J Antimicrob Chemother 61, 962-4.

23. Rodriguez $\mathrm{CH}$, Bombicino $\mathrm{K}$, Granados $\mathrm{G}$, Nastro M, Vay C, Famiglietti A (2009) Selection of colistinresistant Acinetobacter baumannii isolates in postneurosurgical meningitis in an intensive care unit with high presence of heteroresistance to colistin. Diagn Microbiol Infect Dis 65, 188-91.

24. Dejsirilert S, Tiengrim S, Sawanpanyalert P, Aswapokee N, Malathum K (2009) Antimicrobial resistance of Acinetobacter baumannii: six years of National Antimicrobial Resistance Surveillance Thailand (NARST) surveillance. J Med Assoc Thai 92, S34-45.

25. Dizbay M, Altuncekic A, Ergut Sezer B, Ozdemir K, Arman D (2008) Colistin and tigecycline susceptibility among multidrug-resistant Acinetobacter baumannii isolated from ventilator-associated pneumonia. Int $J$ Antimicrob Agents 32, 29-32.

26. Maraki S, Mavros MN, Kofteridis DP, Samonis G, Falagas ME (2012) Epidemiology and antimicrobial sensitivities of 536 multi-drug-resistant gram-negative bacilli isolated from patients treated on surgical wards. Surg Infect 13, 326-31.

27. Montero A, Ariza J, Corbella X, Doménech A, Cabellos C, Ayats J, Tubau F, Ardanuy C, et al (2002) Efficacy of colistin versus $\beta$-lactams, aminoglycosides, and rifampin as monotherapy in a mouse model of pneumonia caused by multiresistant Acinetobacter baumannii. Antimicrob Agents Chemother 46, 1946-52.

28. Marques MB, Brooking ES, Moser SA, Sonke PB, Waites KB (1997) Comparative in vitro antimicrobial susceptibilities of nosocomial isolates of Acinetobacter baumannii and synergistic activities of nine antimicrobial combinations. Antimicrob Agents Chemother 41, $881-5$.

29. Vaara M (1992) Agents that increase the permeability of the outer membrane. Microbiol Rev 56, 395-411.

30. Giannouli M, Popolo AD, Durante-Mangoni E, Bernardo M, Cuccurullo S, Amato G, Tripodi MF, Triassi M, et al (2012) Molecular epidemiology and mechanisms of rifampicin resistance in Acinetobacter baumannii isolates from Italy. Int J Antimicrob Agents 39, 58-63.

31. Ozseven AG, Cetin ES, Aridogan BC, Ozseven L (2012) In vitro synergistic activity of carbapenems in combination with other antimicrobial agents against multidrug-resistant Acinetobacter baumannii. Afr J Microbiol Res 6, 2985-92.

32. Pongpech P, Amornnopparattanakul S, Panapakdee S, Fungwithaya S, Nannha P, Dhiraputra C, Leelarasamee
A (2010) Antibacterial activity of carbapenem-based combinations against multidrug-resistant Acinetobacter baumannii. J Med Assoc Thai 93, 161-71.

33. Linden PK, Paterson DL (2006) Parenteral and inhaled colistin for treatment of ventilator-associated pneumonia. Clin Infect Dis 43, S89-94.

34. Koch-Weser J, Sidel VW, Federman EB, Kanarek P, Finer DC, Eaton AE (1970) Adverse effects of sodium colistimethate: Manifestations and specific reaction rates during 317 courses of therapy. Ann Intern Med 72, 857-68. 